\title{
Italique Italique
}

I| 1998

Varia

\section{Un «roco» sonetto per Veronica. Come nasce il CXXIII delle Rime di Pietro Bembo}

\author{
Lino Pertile
}

\section{OpenEdition}

Journals

\section{Edizione digitale}

URL: http://journals.openedition.org/italique/60

DOI: 10.4000/italique.60

ISSN: 1663-4438

\section{Editore}

Librairie Droz

\section{Edizione cartacea}

Data di pubblicazione: 1 giugno 1998

Paginazione: 9-24

ISBN: 2-600-00239-1

ISSN: 1423-3983

\section{Notizia bibliografica digitale}

Lino Pertile, «Un «roco» sonetto per Veronica. Come nasce il CXXIII delle Rime di Pietro Bembo », Italique [Online], I | 1998, online dal 14 septembre 2009, consultato il 02 mai 2019. URL : http:// journals.openedition.org/italique/60 ; DOI : 10.4000/italique.60 


\section{Lino PERTILE}

UN «ROCO»SONETTO PER VERONICA.

COME NASCE IL CXXIII DELLE RIME

DI PIETRO BEMBO 


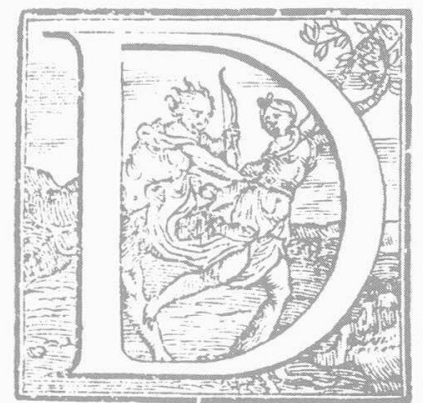

I quindici anni più giovane di Pietro Bembo, Veronica Gambara ne fu una genuina e affezionata ammiratrice. Fu lei probabilmente che, non ancora ventenne, propose al già illustre e maturo Bembo il sonetto da cui ebbe origine de lonh la loro amicizia. Uniamicizia che li accompagnò per tutta la vita, tanto che ancora nel 1544 il vecchio Cardinale le mandava biglietti galanti, e alla morte di lui nel 1547 lei gli dedicava ben due decorosissimi e teneri sonetti. Ciò nondimeno tra $i$ due poeti non vi fu mai vera $e$ propria uguaglianza: il loro rapporto, vissuto all'ombra di Francesco Petrarca, rimase sempre, per riconoscimento reciproco, da maestro ad allieva. Né poteva essere diversamente, date le loro posizioni sociali e le circostanze della loro amicizia.

Se però si considerano $i$ testi poetici che $i$ due si scambiarono, quello che emerge è un quadro più complesso. Veronica si muove non soltanto con maggiore freschezza e franchezza, ma anche con maggiore felicità espressiva del Bembo. Si ha l'impressione che le sue proposte mettano in difficoltà o in qualche modo inibiscano il maestro. O perlomeno questo è quanto a mio avviso emerge dall'episodio che mi appresto a illustrare.

Nel mese di marzo o febbraio del 1530, se non prima, la Gambara mandava al Bembo il sonetto seguente:

A l'ardente desio ch'ognor m'accende di seguir nel camin ch'al Ciel conduce sol voi mancava, o mia serena luce, per discacciar la nebbia che m'offende.

Or, poiché 'l vostro raggio in me risplende, per quella strada ch'a ben far ne induce vengo dietro di voi, fidato duce, che 'l mio voler più oltra non si stende.

Bassi pensieri in me non han più loco; ogni vil voglia è spenta, e sol d'onore e di rara virtù l'alma si pasce,

dolce mio caro ed onorato foco, poscia che dal gentil vostro calore eterna fama e vera gloria nasce. 
Sono versi sereni e lievi, di grande fermezza sintattica e limpida coerenza metaforica. Il campo semantico dell'ardore percorre dall'inizio alla fine, ma discretamente, tutto il sonetto (ardente, accende-spenta, foco, calore: il sole stesso sinsinua nel testo tramite il suo omofono sol ai vv. 3 e 10), ${ }^{2}$ intrecciandosi con quello affine della luminosità e del suo contrario (serena luce-nebbia, raggio, risplende), mentre la grande metafora del camin (seguir, camin, conduce, strada, vengo dietro, duce, più oltra) si sviluppa in parallelo senza nessuno sforzo, con una naturalezza di dettato che riscatta quanto vi ha in essa di inevitabilmente trito. Si noti poi la sequenza desio, voler, voglia, o il gioco sapiente e schietto dei riferimenti personali voi-mia (3), vostro-me (5), voi-mio-me (7-9), e mio-vostro (12-13); o infine l'affettuosa intimità degli stilemi vocativi o mia serena luce $e$ dolce mio caro ed onorato foco. Certo, il tema dell'amore o dell'esempio che nobilita e innalza è topico (anche se qui è la donna che si ispira all'uomo e non, com'è consueto, il contrario); certo, il nume di Petrarca sindovina dietro ogni verso e ogni rima. Per fare qualche esempio, dietro al "camin ch'al Ciel conduce» c'è Rvf. 72, 3 ("la via chial ciel conduce») intrecciato con 14, 6 ("l'amoroso camin che gli conduce»); dietro a "quella strada ch'a ben far ne induce» c'è di nuovo la canzone 72,7 ("la vista ch'a ben far minduce»). ${ }^{3}$ Ma Veronica sa imprimere un certo suo personale frisson (almeno a mio sentire) tra il primo e il secondo verso e tra il penultimo e l'ultimo, proprio dove, in corrispondenza non fortuita con $i$ due maggiori enjambements, il lettore potrebbe aspettarsi altro da quel che poi trova. Il sonetto rivela insomma un'ispirazione tutt'altro che pedestre; allo stesso tempo è un piccolo capolavoro di finezza compositiva, un meccanismo gradevole che funziona a perfezione, anche se confezionato in gran parte con materiali di riporto: non soltanto il Bembo, ma anche il suo severo e schivo consulente in materia di stile, Trifon Gabriele, avrebbe potuto andarne orgoglioso.

Passiamo ora alla risposta per le rime che Pietro Bembo mandava alla Gambara da Padova il primo d'aprile 1530 accompagnandola con queste poche righe di scusa per un ritardo la cui entità ci sfugge (potrebbe trattarsi di giorni, settimane o anche mesi):

Mando a V. S. la mia risposta al vostro leggiadro sonetto; la quale se io ho penato a mandarvi, non è da maravigliarsene tante sono state le sue parti che m'hanno spaventato dal porvi mano. Ma come che sia, nessuna scusa volendo che mi vaglia con voi, nella vostra buona grazia senza fine mi raccomando. Al primo d'Aprile MDXXX. Di Padova. ${ }^{4}$ 
E consuetudine leggere frasi di questo genere come convenevoli privi di sostanza. D'altronde, come si potrebbe credere che il sessantenne Bembo, umanista, editore di Petrarca e di Dante, autore di grandi opere in latino e italiano, degli Asolani, delle Prose, di innumerevoli rime, arbitro della lingua al culmine del suo successo, come credere che un personaggio di tal eminenza penasse o anche soltanto esitasse a buttar giù un semplice sonetto? Certo le sue proteste fanno parte d'un rituale galante. Ebbene, leggiamo il sonetto:

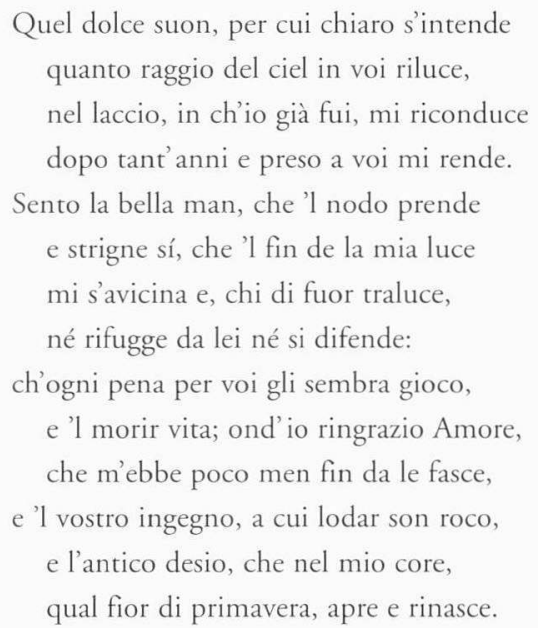

(Rime 123)

Carlo Dionisotti osserva: "La risposta è galante e amichevole insieme, d'una ispirazione perfettamente controllata eppure morbida, fino allo slancio lucido e alto dell'ultimo verso». Tant'è vero che di tanto in tanto sonnecchia anche il buon Omero! Guglielmo Gorni invece considera i tre sonetti certamente rivolti alla Gambara (Rime 63, 64, 123) "tra $i$ prodotti più fiacchi in un genere, il sonetto di corrispondenza, in cui il Bembo diede pur le sue migliori proven; in particolare, ritiene l'ultimo dei tre, cioè quello che qui ci occupa, "stilisticamente dignitoso» ma anche "il più scadente, per la scarsa coerenza metaforica e d'invenzione»; "valga solo - aggiunge Gorni fra pietose parentesi - nella prima quartina, un "suono" che rivela un "raggio" e conduce in un "laccio"»."

In effetti, nel terzo sonetto l'incoerenza delle metafore è tale che avrà fatto raccapricciare Trifon Gabriele, al quale appunto il Bembo aveva dedicato il sonetto precedente, il 122 della raccolta. ${ }^{6}$ Ma anche per altri rispetti la risposta alla Gambara trasuda fatica. Si osservi, per esempio, quel «fin de 
la mia luce", preso di sana pianta da Petrarca Rvf. 18, 6, ma ora adibito a significare, si presume, nientemeno che l'approssimarsi di morte per strangolamento da "bella man". E in che rapporto è "chi di fuor traluce» con il soggetto che dice "io»? Sarà forse la sua anima o il suo cuore, giusta questo o quell' intertesto petrarchesco (Rvf. 147, 13, o 72, 6 o 317, 5, debitamente segnalati da Dionisotti), che, forse a causa della man che strigne il nodo, grottescamente schizza fuori per gli occhi? Gorni rileva e documenta ai vv. 9, 10 e 14 la "ripresa di stilemi caratteristici delle rime asolane". Al v. 9 ("ch'ogni pena per voi gli sembra gioco") ci sarà anche un'eco intrecciata di due versi penitenziali di memoria dantesca, "io dico pena, e dovria dir sollazzo" (Purg. 23, 72) e "che lo salire omai ne parrà gioco» (Purg. 2, 66) con il petrarchesco "et parvi gioco» (Rvf. 133, 7), il quale a sua volta rima con "loco" e più su con "foco" e "roco"; al v. 10 registriamo il vieto ossimoro "e 'l morir vita", seguito a ruota dal prosaico "ond'io ringrazio Amore" che rima con "core" (in risposta facilior a "onorelcalore") e, passando per la zeppa del v. 11 (non sfugga l'inanità di quel "poco men»), fa cumulo trinitario, un po' alla rinfusa, con "[i]l vostro ingegno" e "l'antico desio". Sicché, anche concedendo con Dionisotti uno "slancio lucido e alto" all'ultimo verso, dobbiamo riconoscere che qui la scrittura del maestro si rivela insolitamente inefficace e comunque inferiore a quella dell'allieva; e io non saprei se vedere il sonetto, con Gorni, come un "tentativo di adattamento (...) del mito degli Asolani all'ormai matura Veronica", o non piuttosto come un espediente evasivo, vòlto vuoi a nascondere la mancanza d'ispirazione, vuoi a evitare un confronto troppo personale e impegnativo con la sua corrispondente.

Circa due mesi dopo linvio del sonetto, l'8 giugno 1530, il veneziano Vettor Soranzo, aspirante poeta e affezionato pupillo di Bembo e Messer Trifone, in temporaneo servizio presso la corte pontificia, tra le tante notizie da Roma dava anche qualche ragguaglio al Bembo sulla fortuna del suo ultimo sonetto gambaresco, lamentandosi, come spesso faceva, di non averne ancora avuto copia:

Qui si attende che V. S. risponda al sonetto della Marchesa. Quello a Mad. Veronica è stato tenuto bello di cui mi meraviglio che V. Sig. non mi habbia fatto parte essendo già in bocca d'ognuno.

Questi scambi di sonetti erano una faccenda di pubblico dominio, e i poeti avevano i loro fans che li leggevano e probabilmente ne mandavano a memoria ogni verso. Il Bembo aveva ricevuto due mesi prima tramite il 
Soranzo un sonetto (Ahi quanto fu al mio Sol contrario il fato!) da Vittoria Colonna, Marchesa di Pescara, ${ }^{"}$ e la sua risposta per le rime (Rime 125. Cingi le costei tempie de l'amato), fatta probabilmente nel maggio," non doveva essere ancor giunta a Roma, dove era invece "già in bocca d'ognuno" il sonetto per Madonna Veronica. Se questo fosse veramente "tenuto bello", non possiamo dire; Vettor Soranzo non era certo in una posizione da potersi permettere di dire la verità al suo protettore. Di una cosa si può star sicuri: il Bembo era troppo fine critico per non rendersi conto che quel sonetto alla Gambara gli era venuto proprio male. E dire tutti gli sforzi che gli era costato!

Il codice Barberiniano Latino 2157 della Biblioteca Vaticana raccoglie minute di lettere latine dal Bembo originalmente redatte in veste di segretario di Leone X. La carta che porta il n. 22 (numero presumibilmente bembiano: il codice è privo di numerazione continua), o il $n$. xlix (settecentesco), ci conserva sul verso 10 una copia, autografa del Bembo, di una lettera a Carlo re di Spagna che nella versione a stampa (1538) è datata "XI Calend. Octob. Anno. Quarto. Palo», cioè 1517." La carta è tagliata sotto il testo della lettera al re Carlo, ma nello spazio rimasto tra questa e il taglio sta scritto in senso verticale, di pugno del Bembo, quello che in tutta evidenza è il primo abbozzo del sonetto 123 a Veronica Gambara. ${ }^{12}$ Non sembra che quest'abbozzo risalga tutto a una sola occasione o, per cosí dire, seduta compositiva, visto che penna e inchiostro cambiano dopo un primo segmento di due distici. Anzi mi pare ragionevole supporre che la striscia di carta asportata contenesse, se non il sonetto nella sua forma definitiva, almeno una versione successiva dell'abbozzo.

Ecco dunque i primi quattro versi di questo abbozzo:

Quel dolce stile, ondhom chiaro comprende

Quanto in Donna virtu del ciel riluce

Quel vostro ingegno, che qualunque prende

Cosa a fornir, tanto a lieto fin conduce

Su questi versi si registrano le seguenti aggiunte interlineari: sopra ciel viene inserito raggio; sopra qualunque prende Cosa si prospetta come forma alternativa [qua]ntunque ap. [prende] per.

Si tratta ovviamente di due incipit alternativi, non estranei forse a Petrarca Rvf. 120, 1-2, "Quelle pietose rime in ch'io m'accorsi / di vostro ingegno et del cortese affecto", versi adattissimi alla presente contingenza. 
Nel primo distico la memoria petrarchesca del "dolce stile» (Rvf. 332, 3) e del "vario stile» (Rvf. 1, 5) non è sufficiente a far decollare il sonetto, anche se il secondo verso già presenta quella che poi diverrà la sua struttura definitiva. Ancor meno fecondo di sviluppi il secondo distico che cade tutto e subito, se non per Quel vostro ingegno che verrà buono più tardi al $v$. $12 ;^{13}$ ma intanto ha un effetto scoraggiante sul poeta che, io credo, abbandona la seduta. Evidentemente il Bembo si trova in difficoltà fin dall'inizio: la prima quartina del sonetto di Veronica ha una compattezza, una fluidità, uniarmonia che per ora lui non riesce a emulare.

Quando riprende il lavoro, il Bembo butta giu di seguito ben quattordici versi, o frammenti di versi, tutti segnati dall'iniziale maiuscola, alcuni con forme alternative o varianti interne. Ma sono quattordici versi che non fanno un sonetto. Si tratta, infatti, di una serie continua di prove successive sugli stessi versi 1-7, che si presentano precisamente in quest ordine: $1,2,3,4,3,4,5,6$, $7,3,4,5,6,7$. Il primo distico rappresenta un terzo tentativo di incipit:

Quel vostro dolce suono, in cui s'intende

Quanto in Donna del ciel raggio riluce

Del primo esperimento rimane il deittico d'attacco Quel, favorito dal Petrarca, e l'idea; inoltre si raffina e armonizza il secondo verso col rifiuto dell'originale virtu e l'importazione definitiva di raggio che allittera con riluce e forma con Quanto un bell'iperbato sottolineato dalla cesura. Bembo dev'esserne contento, tant'è vero che per ora non ritorna piu sopra questi due versi, e passa al distico successivo. Più tardi però la durezza prosaica dell'incipit lo lascia insoddisfatto. Elimina dunque vostro, che riapparirà successivamente al $v .12$, e recupera chiaro dalla prima prova: una mossa che lo obbliga a inserire il sintagma in voi a scapito di Donna $e$ a ridisporre le parole al $v$. 2. Con questo risultato definitivo:

Quel dolce suon, per cui chiaro s’intende quanto raggio del ciel in voi riluce

dove si potrebbe pensare, sottomettendosi al sospetto di acutezza, che con la sua ambivalenza semantica l'aggettivo "chiaro" agisca da perno e sottilmente giustifichi la conversione della metafora dal campo del suono a quello della luce: in realtà il passaggio rimane duro.

Del primo emistichio del v. 3 l'abbozzo tenta dapprima due prove: A l'antica pregion $e$ A la rete di pria, subito seguite da una terza, Sotto il giogo primier, che arriva a coprire tutto il $v$. 3 il successivo: 
Sotto 'l giogo primier mi riconduce

Dopo tant' anni, et preso a voi mi rende

Ma Bembo non è soddisfatto e sul margine destro aggiunge una quarta possibilità, Ne gli antichi lacciuo, con cui in effetti esaurisce l'intero repertorio delle metafore piu convenzionali, tradizionalmente adibite a esprimere la condizione dellinnamorato: prigione, rete, giogo, e lacci. Insomma, il vecchio poeta non sa che pesci pigliare, ma almeno il quarto verso gli garba, visto che rimane sostanzialmente immutato fino alla pubblicazione. Segno che a questo punto ha messo a fuoco l'idea che vuole comunicare, cioè il rinascere di Amore, risuscitato dalla poesia di Veronica dopo tant' anni - per la precisione venticinque - se vogliamo con Gorni ritornare ai tempi degli Asolani. Ma andiamo avanti.

Segue un nuovo tentativo sui vv. 3-4:

Mio cor ne primi lacci riconduce

Dopo tant' anni et preso a voi lo rende

Entrano ora nel verso $i$ lacciuoli che prima erano apparsi a margine, mentre oggetto della caccia non è più il poeta in prima persona, ma il suo cuore. La metafora, per quanto letteralmente cruenta (ma c'erano precedenti di indiscussa grandezza) e logicamente incongrua (è ardua impresa prendere un cuore al laccio), dovette piacere per un po' al Bembo. La troviamo infatti subito perseguita in una direzione inattesa nei tre versi successivi:

Sento la bella man, che 'l mio cor prende

Et veggio l'una et l'altra chiara luce

Sfavillar si

Qui le cose prendono dapprima una piega interessante. Il poeta, affascinato dall'immagine del cuore preso nei lacci d'amore e reso alla sua cacciatrice, si lascia andare a una fantasia piuttosto audace, diciamo, francamente sensuale. L'atto di consegna del cuore, appena descritto, viene rivissuto au ralenti e collocato in primo piano dal proprietario legittimo dell'organo. Ora il poeta "sente" e in pari tempo vede, cioè, in una parola, esprime la sensazione della mano di lei che prende il suo cuore e - potenza dell'enjambement! - lo strigne. Quella bella man, già portagli "ignuda" in Rime 27, 8 e da lui "tocca" in sogno a Rime 90, 6 (altri due sonetti del primo decennio del '500), non aveva mai fatto nulla di tanto 
conturbante. Certo non l'aveva fatto nel primo sonetto del ciclo petrarchesco, cosiddetto "del guanto": "O bella man, che mi destringi 'l core / e 'n poco spatio la mia vita chiudi" (Rvf. 199, 1-2); né nel secondo: "Non pur quell' una bella ignuda mano, / ... I ma l'altra et le duo braccia accorte et preste / son a stringere il cor timido et piano" (Rvf. 200, 1-4). Mentre in Petrarca c'era contemplazione e distanza, in Bembo c'è contatto diretto, sensazione: Sento la bella man ... Il Bembo s'è insomma lasciato andare e ha vergato di getto l'unica fantasia viva e sua propria dell'intero sonetto: peccato che non c'entri nulla con la proposta spirituale di Veronica! E soprattutto che smentisca l'icona devota, da lei con tanta classe dipinta, di un Bembo serena luce, fidato duce, onorato foco. Ecco dunque che il poeta si risveglia dal suo sogno erotico, corre ai ripari, colpisce con un tratto di penna il sintagma traditore, e nell'interlinea, sopra il colpevole et strigne, verga un innocente timida. Ora la bella mano non afferra più e decisa stringe, come chi sa quel che vuole, ma avanza esitante, dubitosa, quasi controvoglia. La figura della donna si avvia a rientrare magicamente nei ranghi dell'amor platonico, ridiventa astrazione, letteratura. ${ }^{14}$ Ma forse in quel poco che tuttavia rimane della sua originale fantasia il poeta avverte ancora qualcosa di troppo allusivo, un'ombra di carnalità non interamente rintuzzata: dopotutto, nel Petrarca, «timido» era il cuore e non la mano! Cade perciò anche il timida e con esso ogni senso d'una man che agisce di testa propria ai limiti della decenza. I versi 5-6 prendono ora questa forma:

Sento la bella man, che 'l mio cor prende

Et veggio l'una e l'altra chiara luce

Sfavillar si

Purtroppo, quel saltare di palo in frasca, cioè dal sentire al vedere e dalla mano alla sfavillante luce che in realtà è un occhio (piuttosto bizzarro al singolare), non alletta né convince. Anzi, il verso 6 risulta talmente insipido e trito, talmente condizionato dalla rima luce (per di più già tutta scontata dal banalissimo chiara) che al poeta manca il coraggio di continuare. Il sonetto non vuol venire, e quel poco che viene, viene male. Il Bembo - ci sia concesso ancora per un po' di inseguirne i pensieri sulla carta - si rende conto che, eliminando l'immagine dello "strignere", ha soppresso l'unico frammento vivo della sua invenzione. Deciso a recuperarlo, ma senza cadere nella trappola della sensualità, riparte da capo prendendo ora la rincorsa dal verso 3: 
Ai primi lacci il cor mio riconduce

Dopo tant' anni, et preso a voi lo rende.

La bella et mane accortamente il prende

Et strigne si ch(e)'l fin de la mia luce

Veggo vicina:

Poco da osservare sui versi 3-4: oggetto grammaticale dell'operazione condotta, non si dimentichi, dal dolce suon della poesia di Veronicarimane sempre il cor, ma l'ordine sintattico dei complementi appare ora invertito rispetto alla versione precedente, mentre resta intatto fin dalla prima minuta riconduce, autorizzato da Petrarca Rvf. 194, 10 "ch'Amor per forza a lui mi riconduce». E veniamo ai versi cruciali: il 5 e il 6. Come prima mossa Bembo elimina Sento, troppo personale nel contesto, anzi quasi indecente nell'ambito di un rapporto platonico fra persone attempate. Poi però si fa trascinare da Petrarca Rvf. 23, 149 ("quella fera bella e cruda" che suggestivamente si bacia in rima con "ignuda", stilema già ripreso due volte dal Bembo in un suo altro sonetto anteriore al 1510, il 33, 1-2), e scrive di getto La bella et cruda mano; purtroppo il verso, che deve per forza terminare in prende, non gli viene, finché non lo soccorre, ispirato dall'appena visto Petrarca Rvf. 200, 3 ("duo braccia accorte et preste»), l'avverbio accortamente, che lo obbliga a ritornare sui suoi passi e a depennare cruda. Ora la bella mano, che per un momento aveva potuto sembrare timida, prende il cuore del poeta (chi se lo sarebbe aspettato?) accortamente, cioè con la sagacia, l'avvedutezza o addirittura l'astuzia necessaria a sventare, mettiamo, "una leggiadra vendetta" d'Amore, giusta Petrarca Rvf. 2, 13. Lo prende Et strigne si che'l' fin de la mia luce Veggo vicina. Nell' interlinea sopra Veggo inserisce Sento (ora libero dall'impegno col v. 5), forse allo scopo di evitare un calco troppo smaccato di Petrarca Rvf. 18, 5-6 ("i che temo del cor che mi si parte, / et veggio presso il fin de la mia luce»). L'onore dunque è salvo, ma il ridicolo incombe. Perché, che cosa si riduce a fare ora la bella mano? Prende il cuore e, si badi bene, con accortezza lo stringe a tal punto che il poeta vede $o$, peggio, "sente" avvicinarsi la fine della sua luce, ovvero della sua vita: insomma sta per essere soffocato. Ma qui il Bembo si accorge forse che il suo adattamento di Petrarca Rvf. 18 sconfina nella parodia, e perciò, desolato $e$ (immagino) depresso, pianta l'abbozzo e, dopo tre prove sui vv. 1-2, tre sui vv. 3-4 e due sui vv. 5-6 e l'inizio del v. 7, di nuovo si arrende. Da una parte della sua scrivania il sonetto della Gambara continua a guardarlo in tutta la sua serena compiutezza; dall'altra c'è la somma dei suoi tentativi di risponderle: 


\section{Lino Pertile}

Quel dolce stile, ondhom chiaro comprende

raggio

Quanto in Donna virtu del ciel riluce

ntunque ap.

Quel vostro ingegno, che qualunque prende

per

Cosa a fornir, tanto a lieto fin conduce

Quel vostro dolce suono, in cui s'intende

Quanto in Donna del ciel raggio riluce

A l'antica pregion A la rete di pria Ne gli antichi

lacciuo

Sotto'l giogo primier mi riconduce

Dopo tant'anni, et preso a voi mi rende

Mio cor ne primi lacci riconduce

Dopo tant'anni et preso a voi lo rende

Sento la bella man, che 'l mio cor prende

Timida

Et veggio l'una et l'altra chiara luce

Sfavillar si

Ai primi lacci il cor mio riconduce

Dopo tant'anni, et preso a voi lo rende.

La bella et mane accortamente il prende

Et strigne si chl fin de la mia luce

Sento

Veggo vicina:

È del tutto probabile che, tra questo abbozzo e la versione poi data alle stampe, il sonetto sia passato attraverso altri stadi. In mancanza di una documentazione completa ci dobbiamo accontentare di confrontare l'ultima versione dei $v v$. 3-7, appena esaminati, con quella definitiva. La novità più cospicua consiste nell'estromissione del cuore, il muscolo delicato, palpitante e compromettente che in fase di prova aveva dato tante pene al Bembo. Questa dunque la forma definitiva dei versi 3-4 (il soggetto è sempre il "dolce suon" del sonetto di Madonna Veronica):

nel laccio, in ch' io già fui, mi riconduce

dopo tant' anni e preso a voi mi rende. 
Mentre il cuore viene trasferito in zona meno critica, ciò̀ nel penultimo verso ("e l'antico desio, che nel mio core, I qual fior di primavera, apre e rinasce»), il "dolce suon" agisce ora direttamente sul poeta e lo "riconduce» (unico materiale inderogabile è il petrarchesco) non più ai primi lacci, ma "al laccio», singolare e antonomastico, «in ch' io già fui». Trapiantato il cuore, era ancora necessario evitare ogni riferimento troppo preciso ai primi innamoramenti che risalivano ben oltre i tempi degli Asolani, quando Veronica era ancora adolescente: «in ch' io già fuì è la trovata che risponde a questa esigenza e contribuisce sensibilmente a rendere tutto il discorso più vago e generico, meno personale e impegnativo.

Il cambiamento più decisivo avviene però nella seconda quartina. Caduto il cuore, che cosa fa la bella man, che cosa prende e strigne che il poeta può "sentire" senza imbarazzare la sua non piu giovane corrispondente $e$ rinfocolare inopportunamente la sua fama di uomo non alieno dai piaceri dei sensi? "[I]l nodo", naturalmente, che deve però essere scorsoio ove si debba con esso effettuare uno strangolamento, per quanto metaforico. Con il qual nodo si completa, dopo quella esperita dalla bella mano, la rivisitazione di tutta l'attrezzatura (laccio, cuore, stringere, primi amori, nodo) già messa in opera al tempo delle rime asolane nella canzone Gioia m' abonda:

Né fia per tutto ciò, che quella voglia, che con sí forte laccio il cor mi strinse, quando primieramente Amor lo vinse, rallenti il nodo suo, non pur discioglia, mentre in pié si terrà questa mia spoglia;

(Rime 73, 19-24)16

attrezzatura che riemerge ora come segue: in ordine sparso, in confezione ambigua e neutra, totalmente spersonalizzata anche rispetto alle combinazioni segretamente abbozzate in laboratorio:

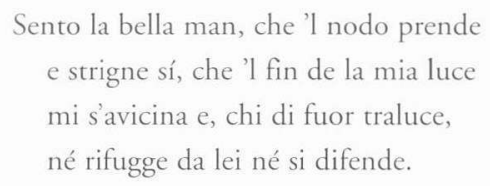

Parafrasando: il poeta sente la bella man che prende il nodo del laccio, si presume, e lo stringe a tal punto che il petrarchesco fin della sua luce, cioè la morte, gli si avvicina, mentre l'altro incerto individuo petrarchesco, cioè chi di fuor traluce (in cui forse si occulta il sopra conculcato cuore, a meno che non sia l'anima), non rifugge né si difende da lei, cioè dalla mano. 
Insomma, l'amore sta soffocandolo, ma il poeta - se in ultima analisi è lui chi di fuor traluce - non muove un dito per salvarsi. Il suo "sentire" si è fatto ora del tutto inverosimile, ma l'importante è che $i$ versi siano purgati di ogni sensualità, di ogni troppo privata allusività, direi quasi di ogni significato. Cosi si difende il Bembo, inibito da una franchezza che lo mette in difficoltà e lo imbarazza. Il suo ricorso a temi e stilemi caratteristici degli Asolani è una strategia evasiva: gli permette di rispondere per le rime senza dir nulla.

Lino Pertile 
1. Ricavo queste notizie da Pietro Bembo, Prose e rime, a cura di C. Dionisotti, Torino, 1960, nota a Rime 60, p. 558. I due sonetti della Gambara in morte del Bembo si leggono ora in VeronICA GAmbarA, Le Rime, a cura di A. Bullock, Firenze-Perth, 1995, pp. 164-65 (nn. 63-64). I riferimenti seguenti saranno a queste edizioni.

2. Devo questa osservazione a Pier Massimo Forni.

3. Per il Canzoniere di Petrarca mi servo dell'edizione commentata a cura di M. Santagata, Milano, 1996.

4. Pietro Bembo, Lettere, edizione critica a cura di E. Travi, vol. III (1529-1536), Bologna, 1992, lettera n. 1072 , p. 122.

5. G. Gorni, Veronica e le altre: emblemi e cifre onomastiche nelle Rime del Bembo, in Veronica Gambara e la poesia del suo tempo nell'Italia settentrionale. Atti del Convegno, Brescia-Correggio, 17-19 ottobre 1985, a cura di C. Bozzetti, P. Gibellini, E. Sandal, Firenze, 1989, pp. $37-57$ (pp. 52-53).

6. Come si può vedere dal suo commento alla Divina Commedia, il Gabriele era particolarmente attento all'uso delle metafore: cfr. metafora e rispondentie nell'indice della mia recente edizione (Annotationi nel Dante fatte con M. Trifon Gabriele in Bassano, edizione critica a cura di L. Pertile, Bologna, 1993).

7. Lettere da diversi re e principi e cardinali e altri uomini dotti a Mons. Pietro Bembo scritte, ristampa anastatica dell'ed. Sansovino, 1560, a cura di D. Perocco, Bologna, Forni, 1985, p. 110 (n. V, 45 dell'edizione originale).

8. Vedi la lettera del Bembo a Vettor Soranzo datata 9 aprile 1530, in Lettere, n. 1078, p. 126. Il sonetto della Colonna ivi menzionato è il 71 dell'ed. A. Bullock, Bari, 1982, p. 38; il Bembo lo giudica «bello e ingenioso e grave, piú che da donna non pare sia richiesto».

9. Cosi Dionisotti in nota al testo del sonetto bembiano in Prose e rime cit., p. 609.

10. La carta era stata in origine una lettera, scritta (presumibilmente) al Bembo il 5 settembre 1527 , in cui Girolamo Foscari lo pregava di intervenire presso il magistrato Angelo Gabriele a favore di un Messer Benedetto "optimo et domestico suo [del Foscari] Amico». Più tardi il Bembo riciclò quella carta utilizzandone il verso per trascrivervi il testo della lettera che voleva includere nella sua raccolta di Brevi. La parte tagliata è quella superiore che senźaltro conteneva il nome del destinatario.

11. È la lettera n. 25 del libro XIII di PIETRO BembO, Epistolarum Leonis Decimi (...) libri, Lugduni, apud heredes Simonis Vincentij, 1538, p. 321.

12. Questa descrizione della carta e la trascrizione dell'abbozzo (del tutto fedele: mi limito a sciogliere le poche abbreviature) si basano su appunti da me presi in Vaticana nel 1986.

13. Di «alto ingegno» parla, dantescamente (vedi Inf. 2, 7), anche il secondo sonetto alla Gambara (64, 7), nonché il 70 a ignota ("Poi che 'l vostr' alto ingegno»), dove ricorre il tema dell'onore presente a sua volta in 63 e 64 . Forse non è dunque troppo avventato (ma vedi in proposito Gorni) ipotizzare che dietro l'ignota del 70 ci possa essere Veronica Gambara.

14. D'altronde, anche a monte del "sogno" erotico si trova forse il modello della Vita Nova: si pensa ovviamente al sogno in cui Amore tiene "nell'una delle mani» il cuore del poeta e poi lo fa mangiare alla donna, ed ella lo "mangiava dubitosamente», cfr. Dante Alighieri, Vita Nova, 1, 14-17. 


\section{Lino Pertile}

Nell'edizione a cura di G. Gorni, Torino, 1996, pp. 17-20.

15. Per accordare fin con il vicina del verso seguente, il Bembo ha corretto chl scrivendovi sopra la, ma la correzione provoca una frattura sintattica; il segno non è comunque di agevole lettura.

16. Ai versi immediatamente precedenti (15-18) Gorni fa risalire il v. 9 del sonetto per la Gambara, "ch'ogni pena per voi gli sembra gioco"; nei due successivi c'è la rima nascelpasce ripresa dalla Gambara ai vv. 11 e 14. 